«Rien ne se perd! ». Récupérer les déchets au Caire, à Casablanca et à Istanbul

"Nothing Goes to Waste!": Trash collection in Cairo, Casablanca and Istanbul

\title{
Bénédicte Florin
}

\section{(2) OpenEdition}

12 Journals

Édition électronique

URL : https://journals.openedition.org/tc/8018

DOI : $10.4000 /$ tc. 8018

ISSN : 1952-420X

Éditeur

Éditions de l'EHESS

\section{Édition imprimée}

Date de publication : 31 octobre 2016

Pagination : 260-263

ISBN : 9782713225291

ISSN : 0248-6016

\section{Référence électronique}

Bénédicte Florin, « «Rien ne se perd! ». Récupérer les déchets au Caire, à Casablanca et à Istanbul », Techniques \& Culture [En ligne], 65-66 | 2016, mis en ligne le 31 octobre 2016, consulté le 29 septembre 2022. URL : http://journals.openedition.org/tc/8018; DOI : https://doi.org/10.4000/tc.8018 


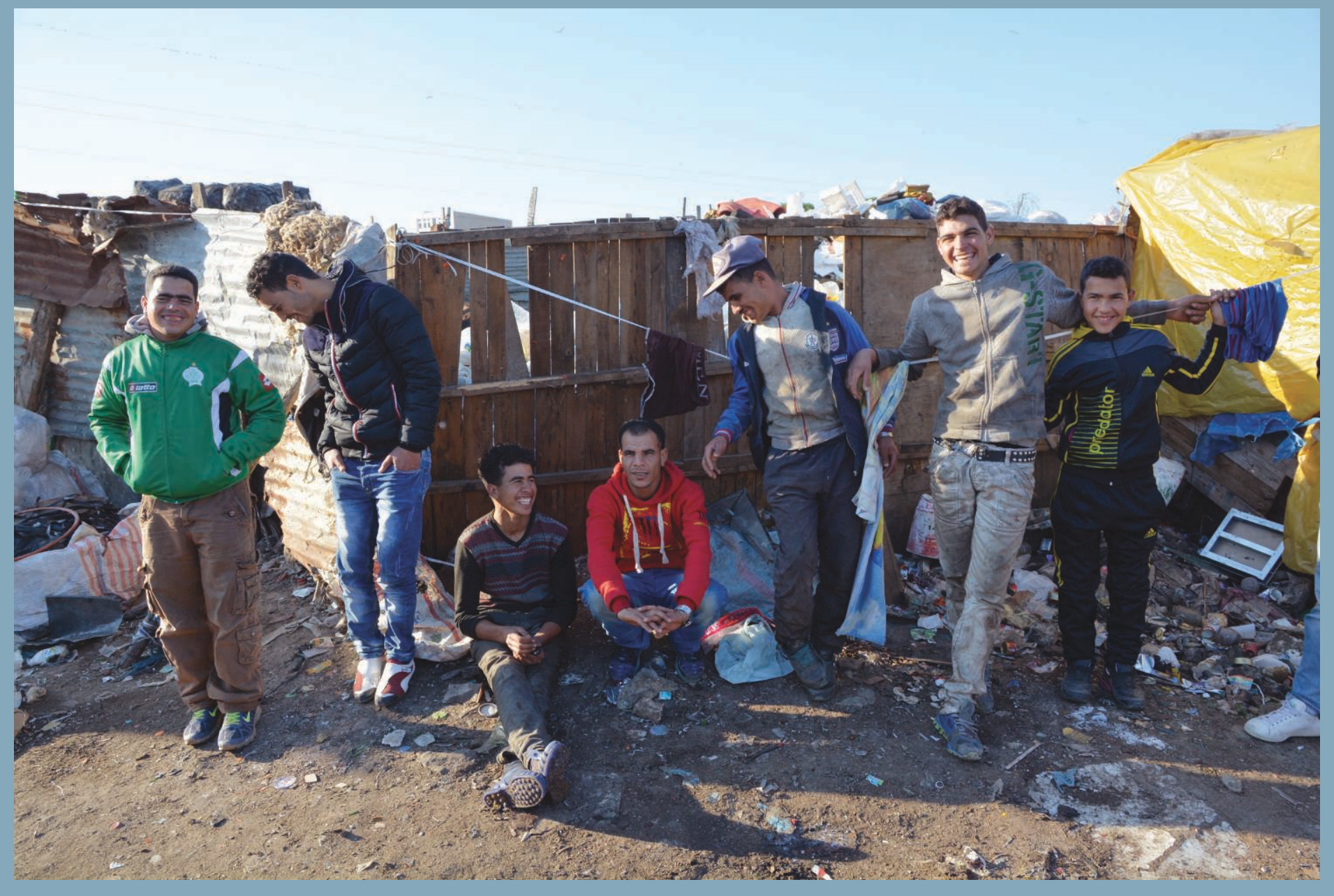




\title{
"Rien ne se perd!" \\ Récupérer les déchets au Caire, à Casablanca et à Istanbul
}

\begin{abstract}
«Rien ne se perd! On contribue à l'économie du pays. C'est grâce à nous que c'est recyclé, sinon tout ça serait brûlé! Ça fait un gain, c'est notre survie et on fait vivre des ouvriers et beaucoup de gens. » (Mustapha, Casablanca, 2013).
\end{abstract}

Les récupérateurs de déchets, zabbâlîn au Caire, bouâra à Casablanca ou toplayıcilar à Istanbul, collectent au porte-à-porte, fouillent dans les poubelles ou les décharges afin de requalifier des restes qui auraient achevé leur trajectoire sans ce travail d'extraction ${ }^{1}$. Pour eux, les rebuts constituent une ressource et un gagne-pain. Non reconnus et stigmatisés, ces travailleurs de l'ombre sont quasi invisibles même si, paradoxalement, très présents dans l'espace public. Ils sont peu audibles et peu conviés aux débats sur l'environnement, l'écologie ou la gestion des déchets, alors même que leur travail de récupération participe au délestage des ordures et à la production de matières premières secondaires.

Face à une exclusion, ressentie comme une injustice, les récupérateurs élaborent pourtant un discours sur leurs compétences professionnelles et sur la dimension environnementale de leur travail. S'ils préfèrent rester invisibles individuellement, ils tentent de s'organiser, de se mobiliser et d'acquérir une visibilité et une légitimité collectives.

Dans ces trois métropoles, les récupérateurs ont toujours été repoussés aux marges et dans les «trous» de la ville: les zabbâlîn cairotes furent souvent expulsés de leurs quartiers; les bouâra trient les déchets dans la décharge de Médiouna et aux confins de Casablanca; les toplayıcılar d'Istanbul rapportent leur collecte chez les grossistes à Süleymaniye et Tarlabaşı, deux quartiers centraux très dégradés. Les activités de tri, pesage, empaquetage, recyclage, s'effectuent dans des espaces interstitiels quasi indécelables et, en ce sens, le travail avec le déchet associe marginalisation spatiale et marginalisation sociale.

Pourtant, les récupérateurs ne forment pas des groupes homogènes: plus on ramasse l'ordure ultime, plus on est en position inférieure, reléguée et réprouvée. Ces gradients du travail sont 
liés au statut du déchet: ceux qui font le sale boulot du «sale boulot» (Hughes 1996 [1962]) sont ceux qui sauvent les déchets de la perte ultime, lors de l'étape ultime dans le lieu ultime qu'est la décharge. À Médiouna, environ 700 personnes collectent jour et nuit dans un monde clos et violent: ils sont à la marge de la marge et dans le reste du reste.

On peut entrer dans le travail après une faillite ou un accident de parcours, mais les généalogies familiales et professionnelles montrent que les chiffonniers le sont souvent de père en fils ou, au Caire, trieuses de mère en fille. Or les parcours professionnels divergent à partir des années 1990 : certains ont pu épargner, acquérir des savoir-faire, embaucher des ouvriers et acheter du matériel et des véhicules. Au Caire, la spécialisation professionnelle, l'ingéniosité des techniques de recyclage, les compétences en termes de commercialisation et d'adaptation à la demande de l'industrie formelle rendent compte de la professionnalisation de ceux qui, enfants, récupéraient la nourriture pour leurs cochons et qui ont pu devenir les businessmen des déchets d'un système que personne n'a vraiment organisé. Certes, dans les trois villes, l'ascension professionnelle n'est pas généralisée, mais elle est possible et se manifeste par les efforts consentis pour la scolarisation des enfants, l'amélioration de l'habitat ou les revendications d'un droit au travail, relevant plus largement d'un droit à la ville.

Au Maroc, en Turquie ou en Égypte, ces dernières décennies ont été marquées par de nombreuses réformes: ainsi, au Caire, l'attribution de la collecte des déchets à des multinationales européennes a bouleversé la corporation des chiffonniers. Ces changements, fondés sur un paradigme modernisateur et un transfert des modèles occidentaux, se matérialisent par de nouvelles techniques auxquelles les récupérateurs tentent de s'ajuster pour conserver l'accès à la ressource: perches pour récupérer dans les containers enterrés, collecte avant le passage des camions-bennes ou négociations avec leurs chauffeurs, accords avec les commerçants pour la reprise de leurs matériaux, etc. Dans les trois métropoles, les récupérateurs élaborent également un discours de légitimation sur leur rôle dans la société et sur le caractère écologique de leur activité. Leur demande de reconnaissance sociale et politique et, dans certains cas, de formalisation du secteur est très forte. Des associations de récupérateurs sont créées, mais restent très inégalement reconnues par les pouvoirs publics. Ces derniers oscillent entre une reconnaissance très partielle et la mise en place de réformes qui rendent la ressource de plus en plus inaccessible.

Finalement, en dépit de ces tentatives d'organisation collective, les récupérateurs restent à la place qui leur est assignée, à savoir à la base du système de plus en plus lucratif et concurrentiel de valorisation des déchets. Leur travail fonde la rentabilité du secteur informel et formel du recyclage, mais cette inclusion ne leur permet pas, ou difficilement, de sortir de la marge. Ainsi que l'écrit D. Lhuillier (2005), il semble bien que perdure, encore et toujours, la superposition de sens construite dans les représentations communes, mais aussi politiques, entre l'« objet déchet » et les « déchets sociaux» que sont les récupérateurs... 


\section{En ligne}

Retrouver l'article complet sur revues. org, TechniquesE Culture 65-66 « Réparer le monde» : http://tc.revues.org/

\section{Notes}

1. Le mot zabbâlîn (sing. zabbal) découle de zibbâla, immondice, ordure; bouâra (sing. bouâr) vient du mot français éboueur; toplayıcilar (sing. toplayıcı) des verbes ramasser, collecter.

\section{I'auteure}

Bénédicte Florin est maître de conférences en géographie à l'université de Tours et chercheuse de l'équipe Monde arabe et Méditerranée, UMR CITERES. Elle a coordonné avec C. Cirelli, Sociétés urbaines et déchets. Eclairages internatio-

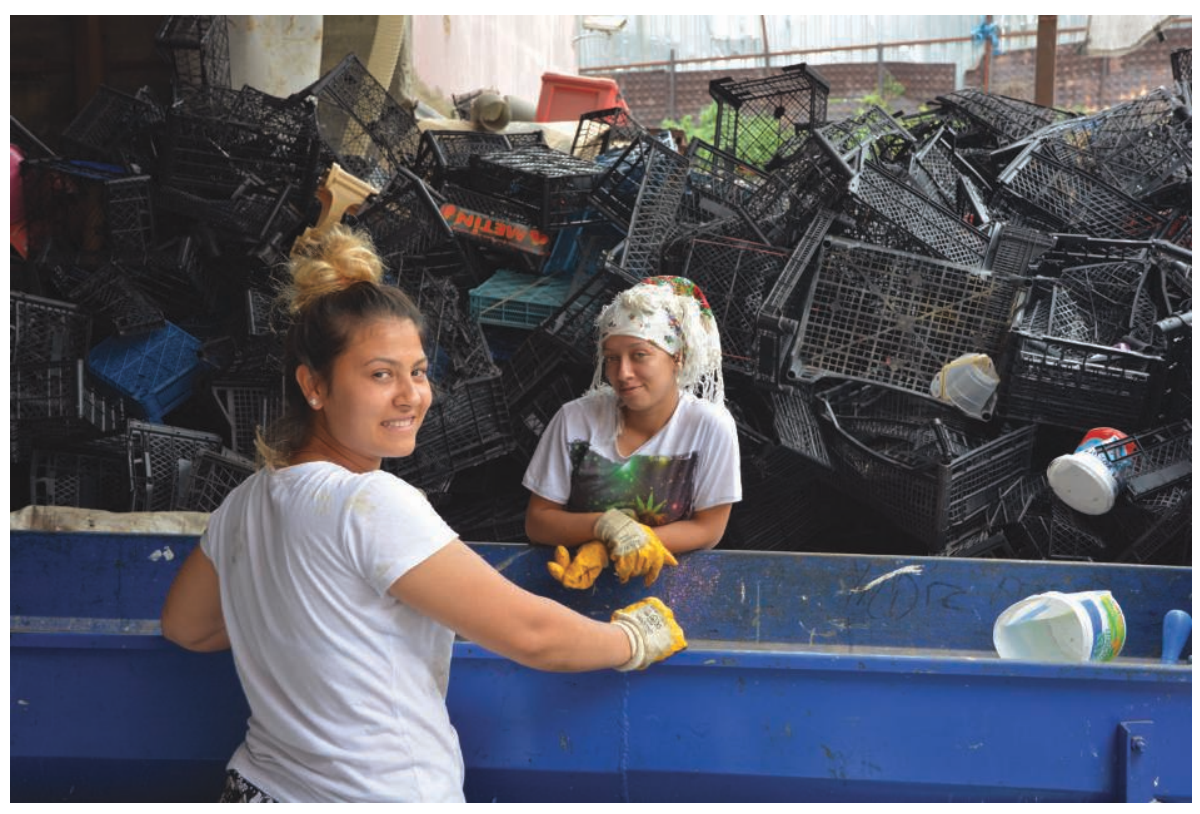
naux, publié aux Presses Universitaires François-Rabelais (2015).

\section{Iconographie}

Image d'ouverture. Jeunes récupérateurs et trieurs à Casablanca. @ P. Garret 2015, Casablanca.

1. (C) P. Garret 2014, Istanbul.

\section{Références}

Hughes, E.-C. 1997 [1962] Le Regard sociologique. Essais choisis. Paris: Éditions de l'EHESS [« Good People and

1. Jeunes femmes au tapis de tri (Istanbul)

Ces jeunes femmes roms de Turquie sont embauchées au tri des plastiques par l'un des plus importants grossistes de Süleymaniye. Anciennes récupératrices dans les rues, elles expliquent qu'elles préferrent leur travail actuel.

\section{Pour citer cet article}

Florin, B. 2016 « "Rien ne se perd!”. Récupérer les déchets au Caire, à Casablanca et à Istanbul», TechniquesECulture 65-66 «Réparer le monde. Excès, reste et innovation», p. 260-263. 